\title{
Applicability of the Telephone Interview for Cognitive Status (Modified) in a community sample with low education level: association with an extensive neuropsychological battery
}

\author{
Teresa Costa Castanho ${ }^{1,2,3}$, Carlos Portugal-Nunes ${ }^{1,2,3}$, Pedro Silva Moreira ${ }^{1,2,3}$, Liliana Amorim ${ }^{1,2,3}$, \\ Joana Almeida Palha ${ }^{1,2,3}$, Nuno Sousa ${ }^{1,2,3}$ and Nadine Correia Santos ${ }^{1,2,3}$ \\ ${ }^{1}$ Life and Health Sciences Research Institute (ICVS), School of Health Sciences, University of Minho, Braga, Portugal \\ ${ }^{2}$ ICVS/3B's, PT Government Associate Laboratory, Braga/Guimarães, Portugal \\ ${ }^{3}$ Clinical Academic Center - Braga, Braga, Portugal \\ Correspondence to: N. C. Santos, PhD, E-mail: nsantos@ecsaude.uminho.pt
}

Objective: To explore the applicability of a Portuguese (PT) version of the Telephone Interview for Cognitive Status (TICS), with a delayed recall item [modified (M)], here termed TICSM-PT, against an extensive (in-person) battery of neuropsychological instruments, in a sample of older individuals with low educational level and without clinically manifest/diagnosed cognitive impairment.

Methods: Following translation/back-translation and pilot testing in 33 community dwellers, 142 community dwellers aged 52 to 84 years $($ mean $=67.45, \mathrm{SD}=7.91)$ were selected from local health care centres for the study (convenience sampling; stratified age and gender). Cronbach's alpha was used to assess internal consistency, and convergent validity was evaluated through the correlation between TICSM-PT and the Mini Mental State Examination (MMSE), as well as with a comprehensive battery of cognitive instruments. Divergent/discriminant validity was assessed through a battery of psychological instruments. The receiver operating curve was determined for TICSM-PT to classify participants with and without possible indication of cognitive impairment.

Results: TICSM-PT showed a satisfactory internal consistency (Cronbach's alpha $=0.705)$, convergent validity and discriminant validity. TICSM-PT presented a positive association with the global cognitive measures Mini Mental State Examination and the Montreal Cognitive Assessment, and also with most neuropsychological parameters. Receiver operating curve curves presented a sensitivity of $90.6 \%$ and specificity of $73.7 \%$. The area under the curve statistic yielded a threshold score equal or below 13.5 for cognitive impairment.

Conclusion: TICSM-PT is a practical tool for rapid cognitive assessment among older individuals with low educational background. Copyright (C) 2015 John Wiley \& Sons, Ltd.

\footnotetext{
Key words: telephone screening; rapid screening; cognition; psychological morbidity; ageing; education History: Received 19 November 2014; Accepted 15 April 2015; Published online 11 May 2015 in Wiley Online Library (wileyonlinelibrary.com)

DOI: $10.1002 /$ gps.4301
}

\section{Introduction}

In ageing research, the possibility of interactions between factors that impact on cognition has gained interest throughout the years. On this, it has been shown that individuals with more school years seem to present better overall test performance (e.g. Santos et al., 2014) and that cognitive test performance is strongly influenced by cultural factors (e.g. Hedden et al., 2002; Park and
Gutchess, 2002; Christofoletti et al., 2007; Weissberger et al., 2013), with psychological morbidity and/or mood also playing a mediator/effector role across educational groups (e.g. Austin et al., 2001; Santos et al., 2014). However, in the 'front-lines' (clinics), as well as in population-based epidemiological research on cognitive performance throughout ageing, it is often difficult (if not impossible) to apply an extensive battery of tests that allow for an integrative and/or comprehensive 
neuropsychological assessment. Thus, rapid administration of standardised tools has recently raised great interest. Research indicates that cognitive function assessment over the telephone may be as reliable and valid as a traditional face-to-face evaluation, providing a useful/practical approach (for review, see Castanho et al. (2014)). Among other strengths, these instruments allow, for example, screening of large-scale populations and to reach individuals with limited mobility, poor motivation and/or who live in rural areas or at great distances from clinical/evaluation centres.

So far, to our knowledge, no telephone cognitive screening instrument has been applied in the Portuguese population and/or in the Portuguese-Portuguese (PT-PT) language, despite Portugal being one of the most rapidly ageing countries, with estimates indicating that $40 \%$ of its total population will be aged 60 years or over by 2050 (United Nations, 2012), and Portuguese being one of the top spoken languages in the world. Furthermore, the current and forecasted educational attainment of the Portuguese population is similar to the average current and forecasted of that worldwide and thus of interest in population-based ageing studies (although its older population is lower educated than those in North American/Western European countries, it is similar to most other more newly developed and/or developing countries; Samir et al., 2010).

Here, the purpose of the current study was to explore the applicability of the most widely used telephone-based cognitive screening tool, the Telephone Interview for Cognitive Status (TICS), here with a delayed recall item of the Modified version (TICSM), against a broader and detailed (in-person) assessment using a neuropsychological battery, in a cohort of older individuals with low educational background and without clinically manifest and/or diagnosed neuropsychiatric or neurodegenerative disorder and/or cognitive impairment.

\section{Methods}

\section{Description of the instrument}

The TICS assessment (original instrument; Brandt et al., 1988 ) is the most popular instrument among telephone-based rapid screening instruments (Castanho et al., 2014). It was developed (and adapted) from the Mini Mental State Examination (MMSE), the 'gold standard' among rapid screening tests, presenting a high validity and reliability on the screening of cognitive impairment. Albeit modelled after the MMSE, the TICS has less ceiling effects, being recognised as a sensitive cognitive measure for detecting mild cognitive impairment and early Alzheimer's disease (Seo et al., 2011), and to document progressive cognitive decline in population samples (Plassman et al., 1994). The original validation study indicates that the TICSM has a good sensitivity (99\%) and specificity (86\%) for distinguishing demented individuals from those without dementia, as well as cognitively impaired from cognitively normal individuals (Welsh et al., 1993; Plassman et al., 1994). Summarily, it assesses working and recent memory, orientation, attention, sentence repetition, conceptual knowledge and nonverbal praxis (Seo et al., 2011). With the inclusion of a delayed recall task, the TICS-Modified (TICSM, original instrument; Welsh et al., 1993) is a further refinement of the TICS. TICSM is a 13-item test and has a scoring range of zero to 39 (in the original instrument), with higher scores representing better cognitive performance. It comprises the following items: subject's age and telephone number; present date (month, day and year), day of week, and season; counting backwards from 20 to one; 10 -word immediate recall list; subtraction calculation; phrase repetition; stating the country's president and prime-minister names (first and last); word opposite enumeration; and 10-word delayed recall list. Although the education level of the subjects can influence the TICS(M) scores, studies have indicated that scores can be adjusted for this (Brandt et al., 1988; Welsh et al., 1993; Gatz et al., 2002; Dennett et al., 2013).

\section{Translation, back-translation and pilot study}

Based on the original TICS version [(Brandt et al., 1988) and later modifications by Welsh et al. (1993) and de Jager et al. (2003) for the recall item (TICSM)], the instrument was translated to PT-PT by two psychologists and a biochemist with expertise in neurosciences, and then back translated by another psychologist and a linguist to establish the linguistic integrity of the version. Translation and backtranslation were enhanced by multidisciplinary group discussions, and the refined draft was shown to be acceptable in a preliminary pilot survey, with the process resulting in minor modifications. The pilot study was conducted to establish whether the PTPT version of the TICS(M) (hereby referred as TICSM-PT) could be understood by the target participants (community dwellers, 50+years of age) and if it was overall well accepted. A total of $n=33$ community-dwelling older individuals participated in the pilot study. A trained psychologist conducted 
the assessment. After completion of the TICSM-PT, participants were asked to comment on whether any parts of the evaluation were difficult to comprehend and to provide their own insights for culture/language adjustments. Test results (scores) from the pilot cohort were not included in the remaining analysis.

The final version of the TICSM-PT comprised 13 items with a maximum score of 39 points. Minor modifications were introduced to account for cultural and language/sonority specificities. Specifically, item 9 ('Who is the reigning monarch now?') was altered to ask for the President's name ('Who is the President of the Republic now?'). Semantic/language issues were also taken into consideration in terms of verbs and adjectives (item 5 'Please subtract 7 from 100' to 'Now I will ask you to calculate 100 minus 7'; item 8 'What do you call the prickly green plant that lives in the desert?' to 'What is a green plant with thorns which is in the desert?'). As there were many difficulties in understanding word enunciation/overall sentence sonority, a few questions were replaced based on other options suggested by the original authors or those in translated versions in other Latin languages (item 11 'What is the opposite of East?' to 'What is the opposite of generous?'; item 12 'Please say this 'Methodist Episcopal' to 'No ifs, ands or buts'). No changes were made to the scoring system and the rating criteria from the original instrument. The TICSM-PT comprised questions evaluating temporal and spatial orientation (7 points), the recall task of the 10 -word list (10 points), the attention and calculation tasks (subtraction and spell backwards) (6 points), five questions evaluating semantic memory (5 points), a task involving language (repetition of a sentence) (1 point) and a delayed recall of the 10 -word list (10 points).

\section{Sample and data collection for the TICSM-PT study}

Potential participants were directly contacted inperson at the local health care centre in Póvoa de Varzim, Braga and Guimarães (Portugal), while in the waiting area for their annual check-up (sample of convenience, stratified sampling for gender and age). A total of $n=142$ community dwellers agreed to participate. Largely, all Portuguese individuals are automatically attributed a local health care centre at birth. The sample was representative of the Portuguese population with respect to gender (62 male participants, $43.7 \%)$, age $(M=67.5, \mathrm{SD}=7.91$, range: $52-84$ years $)$ and formal years of formal education (median $=4$, range: 0-17). Presently, primary/basic grade school education mainly characterises the middle-aged and older Portuguese population, with low percent scores having completed preparatory, secondary and tertiary levels. The participants were equally distributed between urban (city or city outskirts) and rural (villages) areas. The exclusion criteria for participation included inability to understand informed consent, inability to read or write, choice to withdraw from the study, dementia, diagnosed neuropsychiatric, neurodegenerative disorder and/or clinically manifest/diagnosed cognitive impairment. The study was conducted in collaboration with the participants' general practitioner (s), who provided the clinical information.

After establishing acceptance for the telephone testing, subjects completed the extended neuropsychological battery of tests in an in-person evaluation session. Face-to-face examinations (for the neuropsychological battery) and telephone interviews (for the TICSM-PT) were conducted between May 2013 and June 2014. Specifically, within 1 month after the neuropsychological examination, each participant underwent the telephone interview. So to reduce interviewer and participant bias, both the interviewer and the subject were blind to the previous evaluation scores in the neuropsychological battery. Before beginning the TICSMPT evaluation, the participant was asked if he/she was in a quiet room, and with no pen or pencil or temporal information within easy reach (e.g. a newspaper or a calendar). The TICSM-PT required approximately 7/8 min for administration. All participants completed the instrument. The same psychologist conducted all assessments.

The study was conducted in accordance with the Declaration of Helsinki (59th Amendment), and approval was obtained from the national and local ethics committees. Written consent was provided from all participants prior to participation.

Permission was obtained from the Psychological Assessment Resources, Inc. for the use of the TICS with a delayed recall item (TICSM).

\section{Neuropsychological assessment}

A total of $n=97$ individuals completed the comprehensive face-to-face neuropsychological assessment. Instruments were always applied in the same order. The remaining participants did not sit the entire neuropsychological session due to the long nature of the independent evaluation (the battery required approximately $90 \mathrm{~min}$ for completion), albeit agreeing to and later completing the TICSM-PT instrument applied via the telephone interview. All participants $(n=142)$ completed the MMSE. The face-to-face assessment 
followed previously established methodologies for similar cohorts ((Santos et al., 2014) and neuropsychological tests' references therein), designed to assess a wide range of cognitive abilities. Specifically, attention was assessed with the Digit Span forward test and working memory with the Digit Span backward, from Wechsler Adult Intelligence Scale, Third Edition; verbal fluency with the Controlled Oral Word Association tests (COWAT, letters F-A-S); executive function and processing speed with the Digit Symbol Substitution Test (DSST) from the Wechsler Adult Intelligence Scale, Third Edition III and with the Stroop Word-Colour test and memory storage, retention and recall with the Buschke Selective Reminding Test (SRT). Global cognitive functioning was evaluated with MMSE and the Montreal Cognitive Assessment (MoCA). Subjects were also evaluated regarding memory difficulty complaints with the Subjective Memory Complaints Scale (SMC Ginó et al., 2008) and the retrospective and prospective memory slips in the everyday life with the Prospective and Retrospective Memory Questionnaire (PRMQ; Benites and Gomes, 2007). Personality traits were assessed with the NEO Five-Factor Inventor-20 items (NEO-FFI-20; Bertoquini and Pais-Ribeiro, 2006). The Geriatric Depression Scale (GDS, long-version; Barreto et al., 2003) was administered to assess depressive symptoms, the Perceived Stress Scale (PSS; Cohen et al., 1983) to evaluate everyday stress, and the Anxiety Subscale of the Depression Anxiety Stress Scale (DASS; Pais-Ribeiro et al., 2004) to screen anxiety symptoms. Two trained psychologists conducted the in-person assessment during the pilot study, following guidelines previously established and conjoint training in order to reduce interviewers' bias. Only one psychologist performed the evaluations for the main study.

\section{Statistical analyses}

The internal consistency of the TICSM-PT was assessed with Cronbach's alpha and inter-item correlation matrix. Convergent validity was evaluated through the bivariate correlation between the TICSM-PT and MMSE (both at the total score and at the domain score levels), MoCA (total score), Buschke Selective Reminding Test (consistent longterm retrieval, long term storage, delayed recall and intrusions parameters), Stroop (Words, Colours, Words and Colours parameters), DSST (total score), Digits Span (Forward and Backward parameters), and COWAT (admissible and non-admissible words). Divergent validity was assessed by testing the correlation matrix between TICSM-PT and NEO-FFI scales (neuroticism, extraversion, openness, agreeableness and conscientiousness), DASS-21-anxiety subscale, SMC, PRMQ, GDS and PSS (total scores). The relationships between TICSM-PT and cognitive and psychological dimensions were adjusted for education using partial correlations analyses. A receiver operating curve analysis was conducted in order to evaluate the discriminative ability of the TICSM-PT to distinguish between normal and cognitive impairment (CI). A more conservative cut-off for CI of MMSE total score $<23$ was used as test variable to perform the receiver operating curve analysis of TICSM-PT for classifying participants with and without possible CI. This follows the threshold for a similar population (Santos et al., 2014) and the validation study for the Portuguese population (Guerreiro et al., 1994). All the statistical analyses were performed using SPSS version 22 (IBM SPSS Statistics). Statistical significance was defined at the $p<0.05$ level (two-tailed).

\section{Results}

Table 1 displays the descriptive statistics for the scores on TICSM-PT and MMSE by gender, age and education, and Table 2 displays the descriptive statistics for the neuropsychological tests. A significant association was noted between the TICSM-PT and education $(r=0.564, p<0.001)$, indicating that better performance on the TICSM-PT was associated with higher education level. A negative significant association was found between the TICSM-PT and age $(r=-0.243$, $p<0.001$ ), indicating lower test performance with increasing age.

The instrument revealed a satisfactory internal consistency (alpha $=0.705)$. Only the exclusion of the language item, which had the lowest correlation coefficients (Table 3), could improve the internal consistency of the instrument (Table S1). However, since the magnitude of the increment was not large, the original structure was maintained. Concerning the convergent validity, the TICSM-PT presented a positive relationship with the MMSE total score $(r=0.660, p<0.001)$. At the domain-level, orientation, attention/calculation and delayed recall were significantly associated between tests (Table 4). Interestingly, the TICSM-PT registration score (which was not significantly related between tests) was significantly associated with the attention/calculation and language domains in the MMSE (Table 4). Given that the MoCA is considered to be more sensitive (albeit less specific) than the MMSE, it was also considered in the analysis 
Table 1 Descriptive statistics of the TICSM-PT and MMSE total scores by gender, age and education

\begin{tabular}{llllr}
\hline & & $N(\%)$ & TICSM-PT & \multicolumn{1}{c}{ MMSE } \\
\hline Gender & Men & $62(43.7)$ & $20.9(5.2)$ & $26.7(2.4)$ \\
& Women & $80(56.3)$ & $18.2(5.3)$ & $25.6(3.6)$ \\
Age (years) & $<70$ & $94(66.2)$ & $20.2(5.3)$ & $26.9(2.6)$ \\
Years of & $\geq 70$ & $48(33.8)$ & $18.3(5.4)$ & $25.0(3.6)$ \\
education & $<4$ & $99(69.7)$ & $17.6(4.9)$ & $25(3.3)$ \\
& $\geq 4$ & $43(30.3)$ & $23.4(4.2)$ & $28(1.6)$ \\
\hline
\end{tabular}

Values presented as mean (SD)

MMSE, Mini Mental State Examination; TICSM-PT, Telephone Interview for Cognitive Status Modified-Portuguese-Portuguese

Table 2 Descriptive statistics for the cognitive and psychological tests

\begin{tabular}{lcrr}
\hline & Mean (SD) & Min & Max \\
\hline TICSM-PT & $19.36(5.37)$ & 8 & 34 \\
MMSE & $26.06(3.19)$ & 11 & 30 \\
MoCA & $19.02(5.509)$ & 5 & 30 \\
SRT LTS & $23.67(15.072)$ & 0 & 57 \\
SRT CLTR & $16.27(14.534)$ & 0 & 52 \\
SRT delayed recall & $4.54(3.20)$ & 0 & 12 \\
SRT intrusions & $3.66(4.15)$ & 0 & 19 \\
GDS & $9.19(6.74)$ & 0 & 28 \\
Stroop words & $63.27(21.906)$ & 0 & 108 \\
Stroop colours & $48.42(14.569)$ & 11 & 77 \\
Stroop WC & $28.03(14.281)$ & 0 & 58 \\
DSST & $32.2(16.692)$ & 0 & 78 \\
Digits forward & $7.31(1.859)$ & 3 & 12 \\
Digits backward & $4.3(1.92)$ & 0 & 8 \\
COWAT Ad & $21.13(12.575)$ & 0 & 54 \\
COWAT NAd & $0.58(1.142)$ & 0 & 6 \\
SMC & $6.6(4.287)$ & 0 & 18 \\
PRMQ & $31.33(9.451)$ & 16 & 55 \\
PSS & $18.94(7.068)$ & 4 & 37 \\
DASS & $2.45(2.829)$ & 0 & 15 \\
NEO-FFI neuroticism & $6.31(2.852)$ & 0 & 13 \\
NEO-FFI extraversion & $9.92(2.605)$ & 3 & 16 \\
NEO-FFI openness & $7.85(3.471)$ & 0 & 14 \\
NEO-FFI agreeableness & $10.26(2.054)$ & 4 & 15 \\
NEO-FFI conscientiousness & $12.32(1.656)$ & 7 & 16 \\
& & & \\
\hline
\end{tabular}

TICSM-PT, Telephone Interview for Cognitive Status ModifiedPortuguese-Portuguese; MMSE, Mini Mental State Examination; MoCA, The Montreal Cognitive Assessment; SRT LTS, Selective Reminding Test Long-Term Storage; SRT CLTR, Selective Reminding Test Consistent Long-Term Recall; GDS, Geriatric Depression Scale; Stroop WC, Stroop Word Colour Test; DSST, Digit Symbol Substitution Test; Digit Span Forward; Digit Span Backward; COWAT Ad, Controlled Oral Word Association test Admissible; COWAT NAd, Controlled Oral Word Association test Non-Admissible; SMC, Subjective Memory Complaints Scale; PRMQ, Prospective and Retrospective Memory Questionnaire; PSS, Perceived Stress Scale; DASS, Depression Anxiety Stress Scale; NEO-FFI, NEO Five-Factor Inventory-20.

(Nasreddine et al., 2005). The results indicate a positive correlation between the TICSM-PT total score and the MoCA total score $(r=0.750, p<0.001)$. In addition, significant positive associations were found between the TICSM-PT and most cognitive measures, with the exception of those constituting errors (intrusions on Buschke Selective Reminding Test and nonadmissible words on COWAT) (Table 5). With respect to the psychological measures, the TICSM-PT was significantly associated with PRMQ, DASS (only anxiety subscale), GDS and NEO-FFI (openness dimension). Whereas education level did not impact on the significance of the associations between TICSM-PT and cognitive measures, the correlation with DASS, GDS and NEOFFI (openness dimension) was lost after adjusting for school years (Table 5). The area under the curve statistic (Figure 1) equalled 0.870 (95\% confidence intervals: 79-98\%). The calculated optimal TICSM-PT cut-off score for CI was 13.5. Using this threshold, the sensitivity and specificity were 90.6 and 73.7 respectively. Fifteen participants $(10.6 \%)$ of the total sample were classified below this threshold.

\section{Discussion}

The purpose of the present study was to explore the applicability of a PT-PT version of the TICS(M) in a cohort with low education level, against a large battery of neuropsychological tests. Results indicate a linear correlation between the TICSM-PT and the MMSE with a moderate concurrent validity, despite the distinct administration modalities (telephone versus face-to-face). Thus, the TICSM-PT can possibly be effectively used in place of the MMSE in situations where the TICSM-PT would be more practical to administer. More so, the TICSM-PT exhibited moderate to high correlations with the cognitive instruments administered. In a broad sense, the significant association of the TICSM-PT total score with tests that assess for specific cognitive domains indicates that it overall successfully assesses multiple cognitive domains, yielding an overall cognitive score. Interestingly, the strongest associations were observed between the TICSM-PT total score and tests that evaluate executive function (namely the DSST) and working memory (namely the Digit span backward), which are functions particularly vulnerable to decline due to ageing or dementia (Salthouse, 2010). Nonetheless, associations were also noted for most of other domains, including (verbal) memory and attention measures, which are also of consideration in cognitive ageing.

Although in older individuals (co)morbidities with depressive and anxiety symptoms are frequent, cognitive neuroscience research focusing on the unique and mixed consequences of depression and anxiety on test performance is still surprisingly scarce (Dotson et al., 
Table 3 Correlations, variances and covariances of TICSM-PT domains

\begin{tabular}{|c|c|c|c|c|c|c|}
\hline & $\begin{array}{l}\text { TICSM-PT } \\
\text { orientation }\end{array}$ & $\begin{array}{l}\text { TICSM-PT } \\
\text { registration }\end{array}$ & $\begin{array}{l}\text { TICSM-PT } \\
\text { attention/ } \\
\text { calculation }\end{array}$ & $\begin{array}{c}\text { TICSM-PT } \\
\text { Comprehension }\end{array}$ & $\begin{array}{l}\text { TICSM-PT } \\
\text { language/ } \\
\text { repetition }\end{array}$ & $\begin{array}{c}\text { TICSM-PT } \\
\text { delayed recall }\end{array}$ \\
\hline TICSM-PT & 0.735 & 0.251 & 0.696 & 0.211 & 0.016 & 0.273 \\
\hline $\begin{array}{l}\text { TICSM-PT } \\
\text { registration }\end{array}$ & $0.184^{*}$ & 2.513 & 1.050 & 0.777 & 0.072 & 1.951 \\
\hline $\begin{array}{l}\text { TICSM-PT } \\
\text { attention/ } \\
\text { calculation }\end{array}$ & $0.396^{\star \star}$ & $0.323^{\star \star}$ & 4.198 & 0.950 & 0.123 & 1.203 \\
\hline $\begin{array}{l}\text { TICSM-PT } \\
\text { comprehension }\end{array}$ & $0.191^{*}$ & $0.381^{\star *}$ & $0.360^{\star *}$ & 1.658 & 0.043 & 0.867 \\
\hline $\begin{array}{l}\text { TICSM-PT } \\
\text { language/repetition }\end{array}$ & 0.070 & $0.169^{\star}$ & $0.224^{\star \star}$ & 0.124 & 0.072 & 0.049 \\
\hline $\begin{array}{l}\text { TICSM-PT delayed } \\
\text { recall }\end{array}$ & 0.192 & $0.745^{\star *}$ & $0.356^{\star *}$ & $0.408^{* *}$ & 0.111 & 2.728 \\
\hline
\end{tabular}

${ }^{*} p<0.05$.

${ }^{* *} p<0.0 .1$.

TICSM-PT, Telephone Interview for Cognitive Status Modified-Portuguese-Portuguese.

The top half of the table represents the covariance between the different domains. The bottom half of the table represents the Pearson's correlation coefficients between the different domains of TICSM. The diagonal line represents the variance within each domain.

Table 4 Correlations between the TICSM-PT and the MMSE domains

\begin{tabular}{|c|c|c|c|c|c|}
\hline & MMSE orientation & MMSE registration & MMSE attention/calculation & MMSE recall & MMSE language \\
\hline TICSM-PT orientation & $0.324^{\star *}$ & -0.06 & 0.151 & 0.138 & $0.185^{\star}$ \\
\hline TICSM-PT registration & 0.121 & -0.019 & $0.187^{\star}$ & $0.400^{\star *}$ & $0.389^{\star *}$ \\
\hline $\begin{array}{l}\text { TICSM-PT attention/ } \\
\text { calculation }\end{array}$ & $0.327^{\star \star}$ & 0.114 & $0.458^{\star *}$ & $0.361^{\star \star}$ & $0.380^{\star \star}$ \\
\hline TICSM-PT delayed recall & $0.179^{*}$ & -0.005 & $0.233^{\star \star}$ & $0.358^{\star *}$ & $0.391^{* *}$ \\
\hline $\begin{array}{l}\text { TICSM-PT language/ } \\
\text { repetition }\end{array}$ & $0.206^{*}$ & -0.024 & 0.161 & 0.099 & 0.041 \\
\hline
\end{tabular}

${ }^{*} p<0.05$.

${ }^{* *} p<0.01$

TICSM-PT, Telephone Interview for Cognitive Status Modified-Portuguese-Portuguese; MMSE, Mini Mental State Examination.

2014). On this, the present results, which are novel in exploring the association between these dimensions and telephone-administered rapid screening instruments, indicate that higher levels of trait anxiety and depressive symptoms associate with lower levels of performance on the TICSM-PT. This follows studies showing that negative mood measures associate with worse cognitive outcomes (for example Santos et al. (2014)). In fact, it is recommended that depression must be always examined since it can possibly mimic dementia, or be a precursor of it, or even be co-morbid with it (Kuslansky et al., 2004). Furthermore, in older people, besides manifestations of depression, personality traits can often impact on performance in cognitive tasks. These personality traits seem to be somewhat influenced by cultural and sociodemographic characteristics, as well as by the effort that each individual puts in the resolution of the task (Mortensen et al., 2014). As reported by several authors (e.g. Soubelet and Salthouse, 2010; Graham and Lachman, 2012), but again novel for telephone-based instruments, the present findings corroborate a positive association between cognitive ability and the openness trait measure of personality, which was weakened when controlled for education. Trait openness reflects a sustainable approach toward the types of cognitive activities over a lifetime that have been associated with increased cognitive reserve and reduced risk of mild cognitive impairment and dementia in late life (Schretlen et al., 2010). Additionally, despite the higher prevalence of subjective memory complaints in our sample (evaluated to be greater than $25 \%$ ), no associations were found between this measure and the 
Table 5 Correlations between the TICSM-PT total score and cognitive and psychological dimensions from the neuropsychological test battery

\begin{tabular}{lcr}
\hline & \multicolumn{2}{c}{ TICSM-PT } \\
\cline { 2 - 3 } & \multicolumn{1}{c}{$r^{1}$} & Covariance \\
\hline SRT LTS & $0.544(0.38)^{\star \star}$ & 45.979 \\
SRT CLTR & $0.564(0.401)^{\star \star}$ & 45.983 \\
SRT DR & $0.463(0.303)^{\star \star}$ & 8.318 \\
SRT intrusions & $0.071(0.089)$ & 1.079 \\
MoCA & $0.75(0.729)^{\star \star}$ & 23.177 \\
Stroop words & $0.653(0.443)^{\star \star}$ & 82.948 \\
Stroop colours & $0.64(0.443)^{\star \star}$ & 53.762 \\
Stroop WC & $0.663(0.511)^{\star \star}$ & 54.573 \\
DSST & $0.764(0.672)^{\star \star}$ & 71.973 \\
Digits forward & $0.565(0.453)^{\star \star}$ & 5.927 \\
Digits backward & $0.743(0.647)^{\star \star}$ & 8.235 \\
COWAT Ad & $0.606(0.608)^{\star \star}$ & 42.978 \\
COWAT NAd & $-0.197(-0.06)^{\mathrm{a}}$ & -.971 \\
GDS & $-0.235(-0.138)^{\star \mathrm{b}}$ & -9.007 \\
SMC & $-0.094(-0.149)$ & -2.275 \\
PRMQ & $-0.247(-0.339)^{\star}$ & -13.158 \\
PSS & $-0.118(0.082)$ & -4.717 \\
DASS & $-0.377(-0.257)^{\star \star} \mathrm{a}, \mathrm{b}$ & -6.637 \\
NEO-FFI neuroticism & $-0.05(-0.009)$ & -.819 \\
NEO-FFI extraversion & $-0.093(0.023)$ & -1.401 \\
NEO-FFI openness & $0.461(0.278)^{\star \star} \mathrm{b}$ & 9.210 \\
NEO-FFI agreeableness & $-0.002(-0.039)$ & -.018 \\
NEO-FFI & $-0.169(-0.102)$ & -1.615 \\
conscientiousness & & \\
& & \\
\hline
\end{tabular}

$r^{1}$ is the unadjusted (and adjusted for education level) correlation coefficient.

${ }^{a}$ Non-parametric correlations (Spearman's coefficients) were calculated for variables with non-normal distribution.

${ }^{\mathrm{b}}$ These associations are no longer significant when controlling for education level.

${ }^{*} p<0.05$.

${ }^{* *} p<0.01$.

TICSM-PT score. In fact, the correlation between memory complaints and cognitive performance remains controversial. Studies have demonstrated that memory complaints cannot be taken as a definitive indicator for cognitive impairment since they can reflect depressive disorders and other psychological morbidity aspects (Aguiar et al., 2010). Interestingly, we found a negative association between the TICSM-PT and the PRMQ. This might indicate that individuals with higher performance in the TICMS-PT present fewer difficulties in evoking intentions that must be performed within a short or long period of time and previous events and situations.

Finally, an analysis of the receiver operating curves demonstrated satisfactory discriminant validity with the area under the curve statistic pointing for a threshold score of equal or below 13.5 for possible CI. Of note, however, the appropriate cut-off of a neurocognitive instrument depends on its purpose and the effects of false

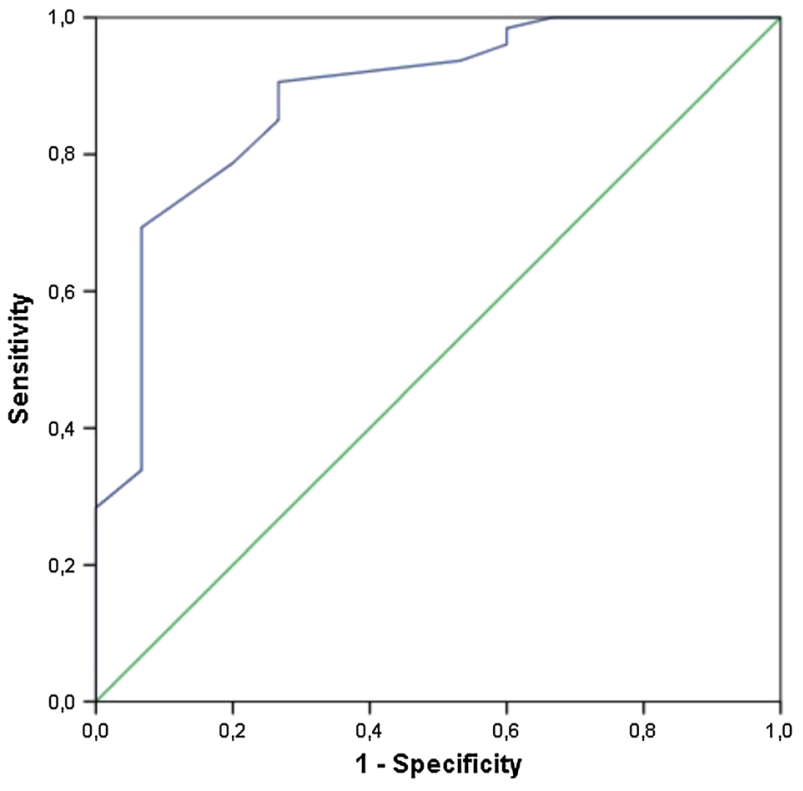

Figure 1 ROC of TICSM-PT-PT. The area under the curve was $87 \%$ (95\% CI: 79-98\%).

positive and false negative results. Here, the determined CI cut-off was both lower than that of the original study (Welsh et al., 1993 reported a mild cognitive impairment threshold of 27 and a sensitivity and specificity of 99 and $96 \%$ respectively) as well as that in other TICSM studies found in the literature (Beeri et al., 2003; Moylan et al., 2004; Knopman et al., 2010; Vercambre et al., 2010). This may be possibly explained when noting that CI cut-off scores can be influenced by the socioeconomic and/or educational characteristics, adding to the discussion on considering appropriate thresholds for the study samples being considered, including in telephone-based instruments (which has not yet been fully addressed, probably due to their more recent use).

This work adds to the research on telephone-based rapid cognitive screening by addressing relevant aspects often missing in similar studies: pilot testing, comparison with an extensive battery of neuropsychological measures, inclusion of a sufficiently large sample for instrument validation and/or applicability/utility studies (' 10 subjects per item' is recommended when examining individual items, although it is less clear if this applies when using the global score), methodological clarity on the validity measures used and, finally, addressing for lower educational settings (vastly similar studies have been conducted in countries with higher educational attainment levels) (for review, see Castanho et al. (2014)). Nonetheless, there are some disadvantages inherent to the telephone assessment that should be addressed and are also of consideration for the present study. These include the limited control over 
environmental factors, the potential impact of hearing impairment and the elimination of the examiner's ability to directly observe the participant's behaviour (for review, see Castanho et al. (2014)). Here, although instructions were given for participants to turn off their televisions or radios and to move any papers or pencils away from where they were, it was not possible to completely control for environmental variables. These may include, for example, the participant referring to a calendar when asked for the date, or the presence of handy notes or of a helpful spouse. Furthermore, the study sample was of convenience and was recruited from the community; therefore, findings may not be extended to a clinical setting and/or be necessarily generalisable. It would also be of interest to (i) assess for performance in individuals that may have already been exposed to telephone assessments, therefore removing any inherent anxiety to the method itself, and (ii) randomise participants to take the TICSM-PT before or after in-person testing. Nevertheless, data adds to previous studies demonstrating that the TICS(M) is a reliable and valid alternative to an in-person evaluation in community-dwelling subjects. More so, it is also of note that in some domains the TICS(M) may be a more difficult test compared to the MMSE and the MoCA. In the TICSM(-PT), the repetition and memorisation of 10 words is required, whereas in the MMSE and in the MoCA, 3 and 5 words are required respectively. Thus, it is here suggested that it may be advantageous to introduce the possibility of repeating the 10 words by the researcher a few times (and/or across trials), which provides the individual with the opportunity to repeat them, also allowing to better investigate the learning process (Knopman et al., 2010). Thus, future studies are needed in order to focus on validating this suggestion and on developing adequate normative data. Here the test administration experience also suggests that certain words were more difficult for participants to distinguish clearly over the telephone and required careful attention to pronunciation. It may be prudent to not only first check the participant's hearing ability with a short pre-test over the telephone before starting the assessment but possibly also throughout the assessment.

\section{Conclusion}

The TICSM-PT demonstrated to be a practical tool for cognitive assessment and a valid method of screening multiple cognitive domains in an older community sample characterised by low education level. The results may have broader implications given that on measures of literacy, (un)employment rates, positive experience/mental health and other sociodemographic characteristics, Portugal ranks close to the Organisation for Economic Co-operation and Development (www.oecd.org/) average. Following this study, future work should address longitudinal assessment and applicability in clinical-based and institutional-based settings and should consider for a randomised sampling.

\section{Conflict of interest}

The research was conducted in the absence of any commercial or financial relationships that could be construed as a potential conflict of interest.

Key points

- The TICS, here with a delayed recall item (modified, M), is one of the most widely used instruments for the screening of cognitive function by telephone.

- The instrument presented associations not only with global cognitive measures but also with a number of cognitive and psychological measures.

- TICSM(-PT) demonstrated to be a practical tool for rapid cognitive assessment and a valid method of screening cognition among older adults with low educational background.

\section{Description of authors' role}

TCC, CPN and NCS recruited the participants. Neuropsychological evaluations were performed by TCC (pilot and main studies) and LA (pilot study). TCC, CPN, PSM and NCS performed the statistical and data analysis. TCC, LA and NCS wrote the first draft of the manuscript. NCS, NS, JAP and TCC conceived and designed the study. All authors participated in data collection and/or interpretation and contributed substantially to the scientific process leading up to the writing of the submitted manuscript, contributed to its writing and have approved of its final version.

\section{Acknowledgements}

This work was funded by the European Commission (FP7): 'SwitchBox' (contract HEALTH-F2-2010259772) and co-financed by the Portuguese North 
Regional Operational Program (ON.2 - O Novo Norte) under the National Strategic Reference Framework (QREN), through the European Regional Development Fund (FEDER). TCC is a recipient of a doctoral fellowship from the Portuguese Foundation for Science and Technology (FCT; SFRH/BD/90078/ 2012), CPN, PSM and LA of research fellowships from the 'MyHealth' project (DoIT-Desenvolvimento e Operacionalização da Investigação de Translação, contract DoIT-13853) and NCS of a 'Switchbox' project post-doctoral fellowship.

\section{References}

Aguiar A, Ribeiro M, Jacinto A. 2010. Subjective memory complaints in the elderly may be related to factors other than cognitive deficits. Dementia e Neuropsychologia 4: 54-57.

Austin MP, Mitchell P, Goodwin GM. 2001. Cognitive deficits in depression: possible implications for functional neuropathology. Br J Psychiatry 178: 200-206.

Barreto J, Leuschner A, Santos F, Sobral M. 2003. Escala de queixas de memória. In Escalas e testes na demência, Grupo de estudos de envelhecimento cerebral e demências (eds).GEECD: Lisboa; 117-120.

Beeri MS, Werner P, Davidson M, Schmidler J, Silverman J. 2003. Validation of the Modified Telephone Interview for Cognitive Status (TICS-M) in Hebrew. Int J Geriatr Psychiatry 18: 381-386.

Benites D, Gomes WB. 2007. Tradução, adaptação e validação preliminar do prospective and retrospective memory questionnaire (PRMQ). Psico-USF 12: 45-54.

Bertoquini V, Pais-Ribeiro J. 2006. Estudo de formas reduzidas do NEO-PI-R: Exame do NeoFFI, do Neo -FFI-R, e do Neo FFI-P para o contexto português. Psicologia: Teoria, Investigação e Prática 11: 85-102.

Brandt J, Spencer M, Folstein M. 1988. The Telephone Interview for Cognitive Status. Neuropsy Neuropsy Be 1: 111-117.

Castanho TC, Amorim L, Zihl J, et al. 2014. Telephone-based screening tools for mild cognitive impairment and dementia in aging studies: a review of validated instruments. Front Aging Neurosci 6: 1-17.

Christofoletti G, Olianni MM, Stella F, Gobbi S, Gobbi LTB. 2007. The influence of schooling on cognitive screening test in the elderly. Dementia \& Neurpyschologia 7: 46-51.

Cohen S, Kamarck T, Mermelstein R. 1983. A global measure of perceived stress. J Health Soc Behav 24: 385-396.

Dennett K, Tometich D, Duff K. 2013. Demographic corrections for the modified Telephone Interview for Cognitive Status. Clin Neuropsychol 27: 1121-1130.

Dotson VM, Szymkowicz SM, Kirton JW, et al. 2014. Unique and interactive effect of anxiety and depressive symptoms on cognitive and brain function in young and older adults. J Depress Anxiety 24: 1-23.

Gatz M, Reynolds CA, John R, et al. 2002. Telephone screening to identify potential dementia cases in a population-based sample of older adults. Int Psychogeriatr 14: 273-289.

Ginó S, Mendes T, Ribeiro F, et al. 2008. Escala de queixas de memória. In Escalas e testes na demência, Grupo de estudos de envelhecimento cerebral e demências (eds). GEECD: Lisboa; 117-120.

Graham EK, Lachman ME. 2012. Personality stability is associated with better cognitive performance in adulthood: are the stable more able? J Gerontol B Psychol Sci Soc Sci 67: 545-554.
Guerreiro M, Silva AP, Botelho MA. 1994. Adaptação à população portuguesa da tradução do Mini-Mental State Examination (MMSE). Revista Portuguesa de Neurologia 1: 9-10.

Hedden T, Park DC, Hisbett R, et al. 2002. Cultural variation in verbal versus spatial neuropsychological function across the life-span. Neuropsychology 16: 65-73.

de Jager CA, Budge MM, Clarke R. 2003. Utility of TICS-M for the assessment of cognitive function in older adults. Int J Geriatr Psychiatry 18: 318-24.

Knopman DS, Roberts RO, Geda YE, et al. 2010. Validation of the Telephone Interview for Cognitive Status - Modified in subjects with normal cognition, mild cognitive impairment, or dementia. Neuroepidemiology 34: 34-41.

Kuslansky G, Katz M, Verghese J, et al. (2004). Detecting dementia with the Hopkins Verbal Learning Test and the Mini Mental State Examination. Arch Clin Neuropsychol 19: 89-104.

Mortensen EL, Flensborg-Madsen T, Molbo D, et al. 2014. Personality in late midlife: associations with demographic factors and cognitive ability. J Aging Health 26: 21-36.

Moylan T, Das K, Gibb A, et al. 2004. Assessment of cognitive function in older hospital in patients: is the Telephone Interview for Cognitive Status (TICS-M) a useful alternative to the Mini Mental State Examination? Int J Geriatr Psychiatry 19: $1008-009$.

Nasreddine ZS, Phillips NA, Bédirian V, et al. 2005. The Montreal Cognitive Assessment, MoCA: a brief screening tool for mild cognitive impairment. J Am Geriatr Soc 53: 695-699.

Pais-Ribeiro JL, Honrado A, Leal I. 2004. Contribuição para o estudo da adaptação portuguesa das escalas de ansiedade, depressão e stress (EADS) de 21 itens de Lovibond e Lovibond. Psicologia, Saúde \& Doenças 5: 229-239.

Park DC, Gutchess AH. 2002. Aging, cognition, and culture: a neuroscientific perspective. Neurosci Biobehav Rev 26: 859-867.

Plassman BL, Newman TT, Welsh KA, Helms M, Breitner JC. 1994. Properties of the Telephone Interview for Cognitive Status: application in epidemiological and longitudinal studies. Neuropsy Neuropsy Be 7: 235-241.

Salthouse TA. 2010. Selective review of cognitive aging. J Int Neuropsychol Soc 16: $754-760$.

Samir KC, Barakat B, Goujon A, et al. 2010. Projection of populations by level of educational attainment, age, and sex for 120 countries for 2005-2050. Demographic Research 22: 383-472.

Santos NC, Costa PS, Cunha P, et al. 2014. Clinical, physical and lifestyle variables and relationship with cognition and mood in ageing: a cross-sectional analysis of distinct educational groups. Front Aging Neurosci 6: 21.

Schretlen DJ, Van der Hulst EJ, Pearlson GD, Gordon B. 2010. A neuropsychological study of personality: trait openness in relation to intelligence, fluency, and executive functioning. J Clin Exp Neuropsychol 32: 1068-1073.

Seo EH, Lee DY, Kim SG, et al. 2011. Validity of the Telephone Interview for Cognitive Status (TICS) and modified TICS (TICSM) for mild cognitive impairment (MCI) and dementia screening. Arch Gerontol Geriatr 52: 26-30.

Soubelet A, Salthouse TA. 2010. The role of activity engagement in the relations between openness/intellect and cognition. Pers Indiv Differ 49: 896-901.

United Nations. 2012. Population Ageing and Development 2012. Department of Economic and Social Affairs. Population Division. http://www.unpopulation.org

Vercambre MN, Cuvelier H, Gayon YA, et al. 2010. Validation study of a French version of the Modified Telephone Interview for Cognitive Status (F-TICS-M) in elderly women. Int J Geriatr Psychiatry 24: 1142-1149.

Weissberger GH, Salmon PD, Bondi MW, Gollan TH. 2013. Which neuropsychological tests predict progression to Alzheimer's disease in Hispanics? Neuropsychology 27: 343-355.

Welsh KA, Breitner JC, Magruder-Habib KM. 1993. Detection of dementia in the elderly using telephone screening of cognitive status. Neuropsy Neuropsy Be 6: 103-110.

\section{Supporting information}

Additional supporting information may be found in the online version of this article. 